\title{
Optimization of rewards in single machine scheduling in the rewards-driven systems
}

\author{
Abolfazl Gharaei, Bahman Naderi and Mohammad Mohammadi*
}

Department of Industrial Engineering, Faculty of Engineering, Kharazmi University, Tehran, Iran

\begin{tabular}{l}
\hline C H R O N I C L E \\
\hline Article history: \\
Received January 20, 2015 \\
Received in revised format 6 \\
February 2015 \\
Accepted 25 March 2015 \\
Available online \\
March 25 2015 \\
\hline Keywords: \\
Optimization \\
Single machine scheduling \\
Rewards-driven systems \\
Delay \\
Earliness \\
Stochastic processing times \\
\end{tabular}
\begin{abstract}
A B S T R A C T
The single machine scheduling problem aims at obtaining the best sequence for a set of jobs in a manufacturing system with a single machine. In this paper, we optimize rewards in single machine scheduling in rewards-driven systems such that total reward is maximized while the constraints contains of limitation in total rewards for earliness and learning, independent of earliness and learning and etc. are satisfied. In mentioned systems as for earliness and learning the bonus is awarded to operators, we consider only rewards in mentioned systems and it will not be penalized under any circumstances. Our objective is to optimize total rewards in mentioned system by taking the rewards in the form of quadratic for both learning and earliness. The recently-developed sequential quadratic programming (SQP), is used by solve the problem. Results show that SQP had satisfactory performance in terms of optimum solutions, number of iterations, infeasibility and optimality error. Finally, a sensitivity analysis is performed on the change rate of the objective function obtained based on the change rate of the "amount of earliness for jobs (Ei parameter)".
\end{abstract}

\section{Introduction}

Manufacturers usually schedule jobs in two steps. In the first step, job processing times are regarded as constant, and a job processing permutation is calculated according to some objectives. In the second step, job processing times are usually compressed, such that the tardiness could be eliminated (Xu et al., 2010). In any company, production scheduling is an essential activity, which aims to prepare a schedule to produce a mix of products as per the production plan of the company. This in turn helps firms improve their productivity. Production scheduling can be classified into the following categories (Panneerselvam et al., 2010).

- Single machine scheduling

- Flow shop scheduling

- Job shop scheduling

- Open shop scheduling

- $\quad$ Batch scheduling

* Corresponding author. Tel \& Fax:+98 (21) 88830891

E-mail address: mohammadi@khu.ac.ir (M. Mohammadi) 
The single machine scheduling problem with single processor or machine consists of single machine to process $n$ jobs. The objective of this problem is to schedule these $n$ jobs on the single machine such that a given measure of performance is minimized. The jobs may be independent or dependent. If the setup times of the jobs are independent of the process sequence of the jobs in the schedule, then the problem is termed as the single machine scheduling problem with independent jobs; otherwise it is termed as single machine scheduling problem with dependent jobs (Panneerselvam et al., 2010).

In the meantime, single machine scheduling problem is one of the most scheduling problems due to its practical importance. The significance of the problem is due to its importance in developing scheduling theory in more complex job shops, and its practical aspects in considering integrated processes as single machine systems. The single machine scheduling has been extensively studied for more than four decades for various performance measures (Baker, 1974, 1995; Conway et al., 1967; French, 1982; Morton \& Pentico, 1993; Pinedo, 2002). As was stated earlier, this problem aims at obtaining the best sequence for a set of jobs in a manufacturing system with a single machine. In other hands, the problem is concerned with finding a sequence among jobs as they proceed through a single machine in order to optimize some performance objectives. In most deterministic scheduling problems job processing times are considered as invariable and known in advance and the objective is to find an optimal sequence that minimizes the expected total weighted number of early and tardy jobs (Kayvanfar et al., 2013). So, a single machine is used to process a set of given jobs at a time. It is assumed that all jobs are executed, simultaneously. It is assumed that all jobs are simultaneously available at time zero and the job can be processed without interruption on the machine. A common due date (d) for all the jobs is given. If a given job is finished after time $d$, a tardiness fine is given; on the other hand, if a given job is finished before time $\mathrm{d}$, an earliness award is presented.

The different measures of performance of the single machine scheduling problem with independent jobs are as listed below (Panneerselvam et al., 2010).

- Minimizing the mean flow time

- Minimizing the maximum lateness

- Minimizing the total tardiness

- Minimizing the number of tardy jobs

Some of the single machine scheduling problem deal with the deterministic case where job attributes (e.g., setup times, processing times, due dates) are known with certainty (Baptiste, 1999; Dauzere et al., 2004; Jolai, 2005; Moore, 1968), but most of single machine scheduling problem deal with the stochastic case in setup times, processing times or due dates. Regarding the consideration of stochastic processing times of jobs, one of attempts was made by Banarjee (1965) for a single machine problem. For a review, the reader is referred to Pinedo et al., (1981), Weiss (1981) and Pinedo (1983). Balut (1973) presents a chance-constrained formulation of a case where processing times are independent normal random variables. Boxma and Forst (1986) study a case where processing times and due dates have identical distributions. Frenk (1991) established a general framework for the single machine stochastic scheduling problems. De et al. (1991) study a case with random processing times and an exponentially distributed common due date. Chunfu (2001) considers a single machine scheduling problem with random processing times to minimize the expected total weighted deviations of completion times from a random common due date. The processing times and the due date are exponentially distributed. Cai and Zhou (2005) consider a case with exponential processing times and random due dates. Naderi and Roshanaei (2014) provide metaheuristic solution methods for No-idle time scheduling of open shops. Jang (2002) and Seo et al. (2005) examined a case where processing times had normal distributions. Several papers have used stochastic processing time in single machine scheduling. Examples include Anderson and Moodie (1969), Cai and Zhou (2007), Portougal and Trietsch (2006), Sarin et al. (1991), Soroush (1999), Soroush and Fredendall (1994), and Wu, Brown, and Beck (2009). As was stated earlier, job processing times are assumed to be fixed and known throughout the entire process. However, empirical studies in several industries have demonstrated that unit costs decline as firms produce more of a product and gain knowledge or experience. For example, Biskup (1999) point out that the worker skills improve by processing of similar tasks repeatedly known as "learning effect”. For extensive reviews about mentioned phrase, the readers can refer to 
Bachman et al. (2004), Biskup (2008) and Janiak et al. (2009). Lee et al. (2010) investigate a singlemachine problem with learning effects with aim to minimize the makespan. Zhang et al. (2010) generalize the model proposed by Cheng et al. (2008) and present the optimal solutions for some single-machine problems. Kuo et al. (2011) study a single-machine problem with the learning effects and the deteriorating jobs to minimize the sum of weighted earliness, tardiness and due-date penalties. Lai et al. (2011) propose a new learning effects model in which the actual job processing time is a general function of the normal processing time of jobs. Yin et al. (2011) present the optimal schedules for some single-machine scheduling problems when both the effects of learning and deterioration were present. Zhu et al. (2011) consider single-machine scheduling problems under the learning effects and resource allocation in a group technology environment. Bai et al. (2012) and Yang (2011) study single-machine scheduling problems with effects of learning and deterioration at same time. Bai et al. (2012) present the optimal solutions for some single-machine problems with exponential learning effects.

As mentioned earlier, our objective is to optimize rewards in single machine scheduling in rewards-driven systems. Rewards-driven systems are critical part of any organization's design. Kind and level of rewards an organization offers influences help organizations to continue to work on this subject (see e.g. Lawler, 1973; Mobley, 1982). Overall, those organizations which give the most rewards tend to attract and retain the most people (Gerhart \& Milkovich, 1992; Adams, 1965). When certain specifiable conditions exist, reward systems have been demonstrated to motivate performance (Gerhart \& Milkovich, 1992; Lawler, 1990; Lawler 1971; Vroom 1964). Rewards-driven systems can reinforce and define the organization's structure (Lawler, 1990). In general, it can be said in rewards-driven systems, operators try to get more reward increase their skills and learning for job earliness. In this paper as was stated earlier, we consider single machine scheduling in rewardsdriven systems in the case that for earliness and learning the bonus awarded to operators and rewards are in the form of quadratic for both learning and earliness. We have considered only rewards in mentioned systems and it will not be penalized for delays or low learning. In this regard, a mathematical model was presented that earliness and learning rewards parameter are in the form of quadratic. For every job, learning and earliness variables configured as zero-one variables in model constraints with aim to find earliness and learning rewards parameter that maximize the total rewards. The main novelties of the paper are as follows:

- The rewards-driven systems in single machine scheduling problem is considered.

- Rewards for both learning and earliness parameters for jobs in single machine scheduling problem and given the quadratic form for learning and earliness parameters for jobs are considered.

- It maximizes the total rewards in single machine scheduling problem aided to provide and solve a quadratic mathematical model. The proposed model contains some constraints which are limited in total rewards for earliness and learning. Mentioned mathematical model is solved aided to SQP algorithm, which is one of the best exact algorithms for optimization and its solution is optimum. It is a powerful and effective class of exact algorithms for a wide range of nonlinear optimization problems.

The rest of this paper is structured as follows: Section 2 shed light on material and method, consist of problem definition and assumptions, notations, mathematical model and solution method. Numerical examples and sensitivity analysis are presented in sections 3 and 4, respectively. Discussion and conclusions are provided in section 5 .

\section{Material and Methods}

\subsection{Problem definition and assumptions}

In this paper, we consider optimization of rewards in single machine scheduling in rewards-driven systems. The proposed study considers awards for earliness and learning the operators. As state earlier, we have considered only rewards in mentioned systems and it will not be penalized for delays or low learning. Our objective is optimization of earliness and learning rewards. In this problem, there are $n$ independent jobs where every job is divided into $j$ activities that must be executed on a machine. Total rewards intended for earliness 
and learning is limited and it has quadratic form. Earliness and learning are independent from each other and due dates are integers. The objective is optimization of earliness and learning rewards while the constraints are satisfied. The following assumptions are used for formulation of the problem:

(1) There are $n$ independent jobs which every job is divided into $j$ activities that must be executed on a machine.

(2) In this scheduling system, the machine is continuously available from time zero.

(3) At any one time only one part of the work on the machine can be run.

(4) In this system, only rewards are considered and it will not be penalized for delays or low learning, total rewards intended for earliness and learning is limited.

(5) Earliness and learning are independent from each other.

(6) Due dates are integers.

(7) Earliness and learning have quadratic form.

\subsection{Notations}

i Index of jobs,

$K \quad$ Index of time periods,

$H \quad$ The number of time periods,

di Due date for job $i$,

Yi Earliness reward parameter for job $i$,

$\mathrm{Zi} \quad$ Learning reward parameter for job $i$,

Ei Amount of earliness for job $i$,

$\mathrm{Li} \quad$ Amount of learning for job $i$,

Pij The computational time for activity $\mathrm{j}$ from job $i$,

Cij The completion time for activity $\mathrm{j}$ from job $i$,

$\mathrm{Pi} \quad$ The total computational time for job $i$,

M The maximum reward is intended to earliness,

$\mathrm{N} \quad$ The maximum reward is intended to learning,

ai $\quad$ Zero and one Variable: If there is earliness, It will be one else zero,

$\beta \mathrm{i} \quad$ Zero and one Variable: If there is learning, It will be one else zero,

Xik Zero and one Variable: If job $i$ is selected in time $k$, It will be one else zero.

\subsection{Mathematical Model}

Referring to problem objective, we consider optimization of rewards in single machine scheduling in rewardsdriven systems which in mentioned systems as for earliness and learning the bonus awarded to operators. The total rewards including earliness rewards and learning rewards, is obtained by:

$$
\mathrm{TR}=\sum_{i=1}^{n} \alpha \mathrm{i} \times \mathrm{Ei} \times \mathrm{Yi}^{2}+\beta \mathrm{i} \times \mathrm{Li} \times \mathrm{Zi}^{2}
$$

In Eq. (1), the first term is the total rewards of earliness and the second term is the total rewards of learning. Note that earliness and learning are independent from each other and $\alpha \mathrm{i}, \beta \mathrm{i}$ are zero and one variables that ensure earliness and learning. As stated earlier in assumptions, there are $n$ independent jobs where every job is divided into $j$ activities that must be executed on a machine and the machine is continuously available from time zero. Total rewards intended for earliness and learning are limited and earliness and learning are independent from each other and finally at any one time only one part of the work on the machine can be run. On this basis, mentioned constraints are:

$$
\left(\sum_{i=1}^{n} \sum_{k=0}^{n} \alpha \mathrm{i} \times\left(\mathrm{di} \times \mathrm{Xik}-\sum_{j=1}^{n} \mathrm{Cij}\right) \times \mathrm{Yi}\right) \leq \mathrm{M}
$$




$$
\begin{aligned}
& \left(\sum_{i=1}^{n} \sum_{k=0}^{n} \beta \mathrm{i} \times\left(\mathrm{Pi} \times \mathrm{Xik}-\sum_{j=1}^{n} \mathrm{Cij}\right) \times \mathrm{Zi}\right) \leq \mathrm{N} \\
& \sum_{\substack{j=1 \\
n}}^{n} \mathrm{Pij} \times \mathrm{Xik}=\mathrm{Pi} \\
& \sum_{i=1} \mathrm{Xik} \leq 1 \\
& \alpha \mathrm{i} \times\left(\mathrm{di} \times \mathrm{Xik}-\sum_{j=1}^{n} \mathrm{Cij}\right) \geq \mathrm{Ei} \\
& \left.\beta \mathrm{i} \times\left(\mathrm{Pi} \times \mathrm{Xik}-\sum_{j=1}^{n} \mathrm{Cij}\right)\right) \geq \mathrm{Li}
\end{aligned}
$$

$$
\alpha \mathrm{i} \times \beta \mathrm{i} \geq 0
$$

Now, the goal is to determine the optimum values of earliness and learning reward parameters for jobs (given in Eq. (1)), such that total reward is maximized. The formulation given in (Eq. (1) to Eq. (8)) is a nonlinearprogramming model. The nonlinear programming characteristic causes the model to be adequately hard to solve by exact methods (Gen, 1997), But since SQP is a powerful and effective class of exact algorithms for a wide range of nonlinear optimization problems (Gill et al. 2010). We will solve mentioned model aided to SQP exact algorithm. Accordingly, in the section 2.4, a sequential quadratic programming method is used to solve this nonlinear programming problem.

\subsection{Solution method}

Sequential (or successive) quadratic programming (SQP) is a technique to solve nonlinear programming (NLP) problems. It is one of the most recently developed and perhaps one of the best methods of optimization. SQP relies on a profound theoretical foundation and provides powerful algorithmic tools for the solution of large-scale technologically relevant problems. SQP has a theoretical basis that is associated with solution of a set of nonlinear equations using Newton's method and derivation of simultaneous nonlinear equations using Kuhn-Tucker conditions to the Lagrangian of the constrained optimization problem. SQP is an iterative procedure that models NLP problems for a given iterate to a quadratic programming (QP) sub-problem, solves that QP subproblem, and then uses the solution to construct a new iterate. This construction is executed in such a way that the sequence converges to a local minimum $\mathrm{X}^{*}$ of the NLP. In general, SQP is a technique for the solution of Nonlinear Programming (NLP) problems with superlinear convergence rate that requires the related QP subproblem must be solvable per iteration (Zhu, 2005). In the next section two numerical examples are provided to illustrate the application of the proposed solution method.

\section{Numerical examples}

Two numerical examples with seven constraints are solved in this section. For instance, the general and the specific data of Examples 1 and 2 are given in Table 1 and Table 2, respectively. In mentioned examples, we suppose, there are 4 jobs and every job is contains of 2 activities. Moreover, the maximum reward is intended to earliness (Parameter $\mathrm{M}$ ) in Example \#1 is 150 and the maximum reward is intended to learning (Parameter $\mathrm{N}$ ) is 180 . The starting point of SQP in the first example is:

$\mathrm{Y}_{1}=1000, \quad \mathrm{Y}_{3}=1000, \quad \mathrm{Z}_{2}=1000, \quad \mathrm{Z}_{3}=1000$ 
The maximum reward is intended to earliness in Example \#2 is 30 and the maximum reward is intended to learning is 40 . The starting point of SQP in the second example is:

$\mathrm{Y}_{1}=5, \quad \mathrm{Y}_{4}=5, \quad \mathrm{Z}_{1}=5, \quad \mathrm{Z}_{3}=5, \quad \mathrm{Z}_{4}=5$

Table 1

General Data for Example \#1

\begin{tabular}{|c|c|c|c|c|c|}
\hline $\mathrm{Ei}$ & $\mathrm{Li}$ & $d_{i}$ & $\mathbf{C}_{\mathrm{ij}}$ & $\mathbf{P}_{\mathrm{ij}}$ & $\mathbf{P}_{\mathrm{i}}$ \\
\hline 2 & 0 & $\mathrm{~d}_{1}=12$ & $\begin{array}{l}\mathbf{C}_{11}=6 \\
\mathbf{C}_{12}=5\end{array}$ & $\begin{array}{l}\mathbf{P}_{11}=6 \\
\mathbf{P}_{12}=7\end{array}$ & 13 \\
\hline 0 & 5 & $\mathrm{~d}_{2}=14$ & $\begin{array}{l}\mathbf{C}_{21}=9 \\
\mathbf{C}_{22}=3\end{array}$ & $\begin{array}{l}\mathbf{P}_{21}=6 \\
\mathbf{P}_{22}=8\end{array}$ & 14 \\
\hline 3 & 3 & $\mathrm{~d}_{3}=10$ & $\begin{array}{l}\mathbf{C}_{31}=4 \\
\mathbf{C}_{32}=2\end{array}$ & $\begin{array}{l}\mathbf{P}_{31}=6 \\
\mathbf{P}_{32}=3\end{array}$ & 9 \\
\hline 5 & 0 & $\mathrm{~d}_{4}=10$ & $\begin{array}{l}\mathbf{C}_{41}=5 \\
\mathbf{C}_{42}=7\end{array}$ & $\begin{array}{l}\mathbf{P}_{41}=5 \\
\mathbf{P}_{42}=9\end{array}$ & 14 \\
\hline
\end{tabular}

Table 2

General Data for Example \#2

\begin{tabular}{cccccc}
\hline $\mathrm{Ei}$ & $\mathrm{Li}$ & $\boldsymbol{d}_{\boldsymbol{i}}$ & $\mathbf{C}_{\mathrm{ij}}$ & $\mathbf{P}_{\mathrm{ij}}$ & $\mathbf{P}_{\mathrm{i}}$ \\
\hline \multirow{2}{*}{5} & 2 & $\mathrm{~d}_{1}=15$ & $\mathbf{C}_{11}=4$ & $\mathbf{P}_{11}=6$ & 12 \\
& & $\mathbf{C}_{12}=6$ & $\mathbf{P}_{12}=6$ & 12 \\
0 & 0 & $\mathrm{~d}_{2}=19$ & $\mathbf{C}_{21}=3$ & $\mathbf{P}_{21}=5$ & \\
& & $\mathbf{C}_{22}=5$ & $\mathbf{P}_{22}=7$ & 8 \\
0 & \multirow{2}{*}{$\mathrm{d}_{3}=14$} & $\mathbf{C}_{31}=6$ & $\mathbf{P}_{31}=4$ & $\mathbf{P}_{32}=4$ \\
\multirow{2}{*}{4} & \multirow{2}{*}{$\mathrm{d}_{4}=14$} & $\mathbf{C}_{32}=2$ & $\mathbf{C}_{41}=7$ & 15 \\
\hline
\end{tabular}

SQP algorithm is applied for solving nonlinear problems, while we have zero and one integer variables in our problem (Variables $\alpha \mathrm{i}$ and $\beta \mathrm{i}$ ). So, first we solve the problem by relaxing binary variables to become continuous variables between zero and one and we round the variables to the colosest values, e.g. zero/one.

The examples have been solved using the SAS 9.2 computer software with its embeded squential quadratic programming. In the next step, we used a simple and logical method for converting continuous variables between zero and one to zero and one integer variables. This means that, we used a sort of LP-Relation to convert a combinatorial problem into a general nonlinear problem and rounded the final solution to get an integer solution. On the basis, we considered the highest values of $\alpha \mathrm{i}, \beta \mathrm{i}$ with 1 value and the lowest values of $\alpha \mathrm{i}, \beta \mathrm{i}$ with 0 value. For instance, in example 1 , there was not highest values for $\alpha \mathrm{i}, \beta \mathrm{i}$, but the lowest value was 0.00053345 which was related to $\beta 2$ variable. Therefore, we considered the zero value for $\beta 2$ and replaced the new value of mentioned variable in constraints. Accordingly, to satisfy the constraints in example 1 , the value of $\beta 3$ variable must be zero. Other variables had integer values of zero or one. So, for this example, the values for $\alpha \mathrm{i}, \beta \mathrm{i}$ are $\beta 2=0, \quad \beta 3=0, \quad \alpha 1=1, \quad \alpha 3=1, \quad \alpha 4=1$. Based on mentioned procedure, the values for $\alpha \mathrm{i}, \beta \mathrm{i}$ for example 2 are $\beta 1=0, \quad \beta 3=1, \quad \beta 4=0, \quad \alpha 1=1, \quad \alpha 4=1$. In continuation of mentioned method, with the new values of the zero and one variables, we rewrote the problem and resolved it aided to SQP algorithm. Table 3 shows the optimal results for Examples \#1 and \#2. It should be noted that problem assumptions are considered in the rewritten model remained without any changes $(\mathrm{M}, \mathrm{N}$ Parameters \& the starting point of SQP and other parameters). According to Table $3, \mathrm{Y}_{\mathrm{i}}$ and $\mathrm{Z}_{\mathrm{i}}$ are optimum solutions which were calculated using SAS software output. Also, $\alpha \mathrm{i}, \beta \mathrm{i}$ are zero and one integer variables. The "objective function value" shown in Table 3 is calculated based on Eq. (1). The number of steps taken by the SQP algorithm to achieve the optimal solution are shown in column "Number of iterations". Since the computation is carried out in finite-precision arithmetic, rounding errors prevent the algorithms from exactly satisfying the preceding condition. Instead, we terminate the algorithms at some small threshold values. These threshold values can be measured in an absolute or relative sense that recent issue is 
shown as the column "Optimality error" in mentioned table. Also we define the relative infeasibility to be the maximum constraint violation in relative measure in column "infeasibility".

Table 3

Optimal results for Examples \#1 and \#2

\begin{tabular}{|c|c|c|c|c|c|c|}
\hline Example No & Optimum solution & $\begin{array}{l}\text { Objective } \\
\text { function value }\end{array}$ & $\begin{array}{l}\text { Number of } \\
\text { iterations }\end{array}$ & Infeasibility & Optimality error & Complementarity \\
\hline Example 1 & $\begin{array}{ll}Y_{1} & 203007.89 \\
Y_{3} & 621153.21 \\
Y_{4} & 10317512.03 \\
\alpha 1 & 1 \\
\alpha 3 & 1 \\
\alpha 4 & 1\end{array}$ & 5.33495E14 & 1873 & 0.0004897212 & 0.9823236879 & 0.0005267859 \\
\hline Example 2 & $\begin{array}{ll}\mathrm{Y}_{1} & 256.34 \\
\mathrm{Y}_{4} & 378.23 \\
\mathrm{Z}_{3} & 305.22 \\
\beta 3 & 1 \\
\alpha 1 & 1 \\
\alpha 4 & 1\end{array}$ & 993941.958 & 1903 & 0 & 0.9836065574 & 0.4887928721 \\
\hline
\end{tabular}

\section{Sensitivity analysis}

A sensitivity analysis on the effect of "amount of earliness for jobs (Ei parameter)" on the optimal result has been performed in this section only for Example \#1 with the initial data shown in Table 1. It involves increasing or decreasing Ei parameter at the rates of $\pm 20, \pm 40$ and \pm 60 percents. Table 4 shows the results. Moreover, Fig. 1 shows the change rate of the objective function with respect to the change rate of the Ei parameter. It can be seen from Fig. 1 that there exist a direct relationship between an increase/decrease in the change rate of the Ei parameter and the change rate of the objective function. Based on Fig. 1, the covariance between change rate of the objective function vs. the change rate of the Ei parameter is 0.15 and correlation coefficient is 0.97 . Note that positive or negative change rates of an equal amount for the Ei parameter results in an almost equal change rates of the objective function. This can be an indicative for the good performance of SQP algorithm.

\section{Table 4}

Effects of Ei on the optimal results of Example \#1

\begin{tabular}{llllll}
\hline $\begin{array}{l}\text { The rate of increase/ } \\
\text { decrease in Ei }\end{array}$ & $\begin{array}{l}\text { Objective } \\
\text { function value }\end{array}$ & $\begin{array}{l}\text { Number of } \\
\text { iterations }\end{array}$ & Infeasibility & Optimality error & Complementarity \\
\hline $1.20 \mathrm{Ei}$ & 94500.0005 & 14 & $6.072645 \mathrm{E}-7$ & $5.8439907 \mathrm{E}-8$ & $6.072645 \mathrm{E}-7$ \\
$1.40 \mathrm{Ei}$ & 103500.00024 & 19 & $9.4792098 \mathrm{E}-8$ & $3.4748325 \mathrm{E}-8$ & $1.5819856 \mathrm{E}-7$ \\
$1.60 \mathrm{Ei}$ & 112500.00091 & 30 & $2.5543272 \mathrm{E}-7$ & $7.4617963 \mathrm{E}-9$ & $2.5543272 \mathrm{E}-7$ \\
$0.80 \mathrm{Ei}$ & 76499.97878 & 42 & 0 & $1.3900388 \mathrm{E}-6$ & $5.8585041 \mathrm{E}-6$ \\
$0.60 \mathrm{Ei}$ & 43031.249533 & 38 & $1.392237 \mathrm{E}-7$ & $1.6579085 \mathrm{E}-7$ & $4.5956429 \mathrm{E}-7$ \\
$0.40 \mathrm{Ei}$ & 28800.000146 & 33 & $3.0976483 \mathrm{E}-7$ & $9.6785716 \mathrm{E}-6$ & $3.0976483 \mathrm{E}-7$ \\
\hline
\end{tabular}

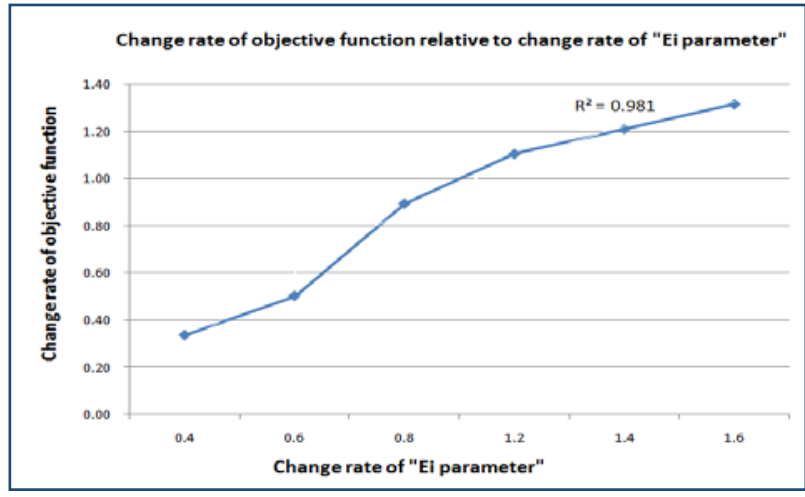

Fig. 1. Change rate of the objective function vs. the change rate of the Ei parameter 


\section{Conclusions and future study}

In this paper, we have considered optimization of rewards for a single machine scheduling in rewards-driven systems. We have considered only rewards in mentioned systems and it will not be penalized for delays or low learning. In mentioned system, there are $n$ independent jobs where every job is divided into $j$ activities that must be executed on a machine and the machine is continuously available from time zero, also total rewards intended for earliness and learning are limited and have quadratic forms. Earliness and learning are independent from each other and finally at any one time only one part of the work on the car can be run. The goal was to determine the optimum values of earliness and learning reward parameters for jobs, such that total reward would be maximized while the constraints were satisfied. The formulation is a nonlinear-programming model which is more applicable for real-world single machine scheduling problems. The recently-developed sequential quadratic programming (SQP), as one of the best optimization method in nonlinear-programming is used for solve the problem. Two numerical examples have been solved to demonstrate the applicability of the proposed methodology and to evaluate its performance. The results show that SQP had satisfactory performance in terms of optimum solutions, number of iterations to achieve the optimum solution, infeasibility and optimality error. At last, a sensitivity analysis on the effect of the Ei parameter on the objective function confirmed excellent performance of SQP optimum algorithm. In this study, we have used a simple and logical method for converting continuous variables between zero and one to zero and one integer variables. The method is suitable for small size problem. Also, it can be used in large size problems, but it is time consuming for solving problems in large size. For future studies, researchers in this field can use other procedures or algorithms to convert a combinatorial problem into a general nonlinear problem which are not time consuming. As a good future research, we recommend adding two parameters as learning probability and earliness probability to problem. We may also consider learning and earliness parameters as fuzzy parameters.

\section{References}

Adams, J. S.(1965). Injustice in Social Exchange. In Berkowitz, L. (Ed.) Advances in experimental social psychology. 2, New York: Academic Press, 267-299.

Anderson, D., \& Moodie, C. (1969). Optimal buffer storage capacity in production line systems. International Journal of Production Research, 7(3), 233-240.

Bachman, A., \& Janiak, A. (2004). Scheduling jobs with position-dependent processing times. Journal of the Operational Research Society, 55(3), 257-264.

Bai, J., Li, Z. R., \& Huang, X. (2012). Single-machine group scheduling with general deterioration and learning effects. Applied Mathematical Modelling,36(3), 1267-1274.

Bai, J., Wang, M. Z., \& Wang, J. B. (2012). Single machine scheduling with a general exponential learning effect. Applied Mathematical Modelling, 36(2), 829-835.

Baker, K.R.(1974). Introduction to Sequencing and Scheduling. John Wiley, New York.

Baker, K.R.(1995). Elements of Sequencing and Scheduling. Dartmouth College, Hanover, NH.

Balut, S. J. (1973). Scheduling to minimize the number of late jobs when set-up and processing times are uncertain. Management Science, 19(11), 1283-1288.

Banerjee, B. P. (1965). Single facility sequencing with random execution times.Operations research, 13(3), 358-364.

Baptiste, P. (1999). Polynomial time algorithms for minimizing the weighted number of late jobs on a single machine with equal processing times. Journal of Scheduling, 2(6), 245-252.

Biskup, D. (1999). Single-machine scheduling with learning considerations.European Journal of Operational Research, 115(1), 173-178.

Biskup, D. (2008). A state-of-the-art review on scheduling with learning effects.European Journal of Operational Research, 188(2), 315-329.

Boxma, O. J., \& Forst, F. G. (1986). Minimizing the expected weighted number of tardy jobs in stochastic flow shops. Operations Research Letters, 5(3), 119-126. 
Cai, X., \& Zhou, X. (2005). Single-machine scheduling with exponential processing times and general stochastic cost functions. Journal of Global Optimization, 31(2), 317-332.

Cai, X., \& Zhou, S. (1997). Scheduling stochastic jobs with asymmetric earliness and tardiness penalties. Naval Research Logistics (NRL), 44(6), 531-557.

Cheng, T. E., Wu, C. C., \& Lee, W. C. (2008). Some scheduling problems with sum-of-processingtimes-based and job-position-based learning effects.Information Sciences, 178(11), 2476-2487.

Jia, C. (2001). Stochastic single machine scheduling with an exponentially distributed due date. Operations Research Letters, 28(5), 199-203.

Conway, R.W., Maxwell, W.L., \& Miller, L.W. (1967). Theory of scheduling. Addison-Wesley, Reading, MA.

Dauzère-Pérès, S., \& Sevaux, M. (2004). An exact method to minimize the number of tardy jobs in single machine scheduling. Journal of scheduling, 7(6), 405-420.

De, P., Ghosh, J. B., \& Wells, C. E. (1991). On the minimization of the weighted number of tardy jobs with random processing times and deadline. Computers \& Operations Research, 18(5), 457-463.

French, S. (1982). Sequencing and Scheduling: An Introduction to the Mathematics of the Job-Shop. John Wiley, New York.

Frenk, J. B. G. (1991). A general framework for stochastic one-machine scheduling problems with zero release times and no partial ordering. Probability in the Engineering and Informational Sciences, 5(3), 297-315.

Gen, M.(1997). Genetic algorithm and engineering design. John Wiley \& Sons, New York, NY, USA.

Gerhart, B., \& Milkovich, G. T.(1992). Employee Compensation: Research and Practice. In M. D. Dunnette and L. M. Hough (Eds.) Handbook of Industrial and Organizational Psychology, Palo Alto, CA: Consulting Psychologists Press, $2^{\text {nd }}$ ed. , Vol. 3, pp. 475-569.

Gill, P.E., \& Wong, E. (2010). Sequential Quadratic Programming Methods. UCSD Department of Mathematics. Technical Report NA-10-03.

Jang, W. (2002). Dynamic scheduling of stochastic jobs on a single machine.European Journal of Operational Research, 138(3), 518-530.

Janiak, A., \& Rudek, R. (2009). Experience-based approach to scheduling problems with the learning effect. IEEE Transactions on Systems, Man and Cybernetics, Part A: Systems and Humans, 39(2), 344-357.

Jolai, F. (2005). Minimizing number of tardy jobs on a batch processing machine with incompatible job families. European Journal of Operational Research, 162(1), 184-190.

Kayvanfar, V., Mahdavi, I., \& Komaki, G. M. (2013). Single machine scheduling with controllable processing times to minimize total tardiness and earliness. Computers \& Industrial Engineering, 65(1), 166-175.

Kuo, W. H., \& Yang, D. L. (2011). A note on due-date assignment and single-machine scheduling with deteriorating jobs and learning effects. Journal of the Operational Research Society, 62(1), 206-210.

Lai, P. J., \& Lee, W. C. (2011). Single-machine scheduling with general sum-of-processing-time-based and position-based learning effects. Omega, 39(5), 467-471.

Lawler, E.E. (1971). Pay and Organizational Effectiveness: A Psychological View. New York: MacGraw-Hill.

Lawler, E. E. (1973). Motivation in Work Organizations. Monterey, CA: Brooks/Cole.

Lawler, E.E. (1990). Strategic Pay: Aligning Organizational Strategies and Pay Systems. San Francisco, CA: Jossey-Bass.

Lee, W. C., Wu, C. C., \& Hsu, P. H. (2010). A single-machine learning effect scheduling problem with release times. Omega, 38(1), 3-11.

Mobley, W.H. (1982). Employee Turnover: Causes, Consequences, and Control, Reading. MA: Addison-Wesley.

Moore, J. M. (1968). An n job, one machine sequencing algorithm for minimizing the number of late jobs. Management Science, 15(1), 102-109.

Morton, T.E., \& Pentico, D.W. (1993). Heuristic Scheduling Systems. John Wiley, New York. 
Naderi, B., \& Roshanaei, V.(2014). No-idle time scheduling of open shops: modeling and metaheuristic solution. International Journal of Supply and Operations Management, 1, 54-68.

Panneerselvam, S., \& Sockalingam, N. (2010). Literature review of single machine scheduling Problem with uniform parallel machines. Intelligent Information Management, 2, 457-474.

Pinedo, M. (1983). Stochastic scheduling with release dates and due dates. Operation Research, 31, 559-572.

Pinedo, M.L.(2002). Scheduling: Theory, Algorithms, and Systems. $2^{\text {nd }}$ Ed. Prentice Hall.

Pinedo, M., \& Schrage, S. (1981). Stochastic shop scheduling: A survey, in: Deterministic and stochastic scheduling. M.A.H. Dempster, J.K. Lenstra and A.H.G. Rinnooy Kan (eds.), Reidel Dordrecht 181-196.

Portougal, V., \& Trietsch, D. (2006). Setting due dates in a stochastic single machine environment. Computers \& Operations Research, 33, 1681-1694.

Sarin, S., Erdel, E., \& Steiner, G.(1991). Sequencing jobs on a single machine with a common due date and stochastic processing times. European Journal of Operational Research, 27, 188-198.

Seo, D.K., Klein, C.M., \& Jang, W. (2005). Single machine stochastic scheduling to minimize the expected number of tardy jobs using mathematical programming models. Computers \& Industrial Engineering, 48, 153-161.

Soroush, H. (1999). Sequencing and due-date determination in the stochastic single machine problem with earliness and tardiness costs. European Journal of Operations Research, 113, 450-468.

Soroush, H., \& Fredendall, L. (1994). The stochastic single machine scheduling problem with earliness and tardiness costs. European Journal of Operational Research, 77, 287-302.

Vroom, V. H. (1964). Work and Motivation. New York: Wiley.

Weiss, G. (1981). Multi server stochastic scheduling, in: Deterministic and stochastic scheduling. M.A.H. Dempster, J.K. Lenstra and A.H.G. Rinnooy Kan (eds.), Reidel Dordrecht 157-180.

Wu, C., Brown, K., \& Beck, J. (2009). Scheduling with uncertain durations: Modeling b-robust scheduling with constraints. Computers \& Operations Research, 36, 2348-2356.

Xu, K., Feng, Z., \& Jun, K. (2010). A tabu-search algorithm for scheduling jobs with controllable processing times on a single machine to meet due-dates. Computers \& Operations Research, 37(11), 1924-1938.

Yang, S. J. (2011). Group scheduling problems with simultaneous considerations of learning and deterioration effects on a single-machine. Applied Mathematical Modelling, 35(8), 4008-4016.

Yin, Y., \& Xu, D. (2011). Some single-machine scheduling problems with general effects of learning and deterioration. Computers \& Mathematics with Applications, 61(1), 100-108.

Zhang, X., \& Yan, G. (2010). Machine scheduling problems with a general learning effect. Mathematical and Computer Modelling, 51(1), 84-90.

Zhu, Z. (2005). An efficient sequential quadratic programming algorithm for nonlinear programming. Journal of Computational and Applied Mathematics,175(2), 447-464.

Zhu, Z., Sun, L., Chu, F., \& Liu, M. (2011). Single-machine group scheduling with resource allocation and learning effect. Computers \& Industrial Engineering, 60(1), 148-157. 